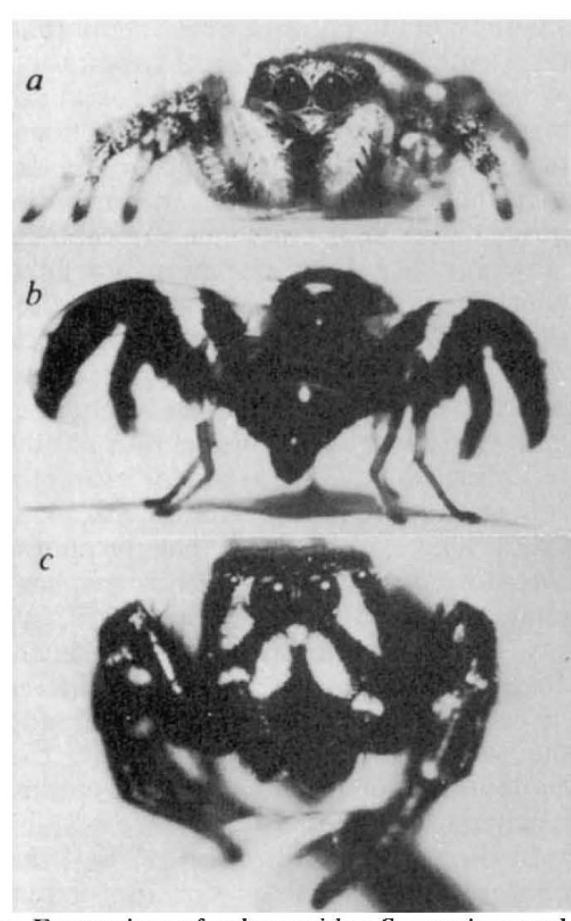

$a$, Front view of zebra spider $S$. scenicus and $b$, posterior view of snowberry fly $R$. zephyria. (Courtesy of M. H. Mather and B. D. Roitberg.) $c$, Male jumping spider $M$. imperialis.

the fly performs its side-to-side dance.

The second paper, by E. Greene, L.J. Orsak and D.W. Whitman (Science 236, $310-312 ; 1987)$, reaches essentially the same conclusions about a New Mexico tephritid Zonosemata vittigera, but these authors took the study rather further. They argue that the real reason for the resemblance between the fly and spider might be because jumping spiders are themselves unpleasant, and that mimicking them would protect the flies against other predators that would normally avoid jumping spiders (Batesian mimicry). They tested tephritids and houseflies with four other predators: an oxyopid spider, an assassin bug, a praying mantis and a whiptail lizard, and found that there is no difference in the acceptability of the two flies, measured as the time taken to capture them. This result contrasts with the situation with jumping spiders, which back away from the tephritids but attack houseflies instantly. Thus, the protection afforded by the tephritid wing pattern and display does seem to be specific to predation by jumping spiders, and other predators are not fooled.

A problem in trying to work out the origins of this evidently successful strategy is that the display that tephritids make to jumping spiders is the same as that used in courtship, according to Mather and Roitberg. Many flies, especially those with patterned wings, have wing-waving displays whose function is to attract the attention of conspecifics, and males often display to anything that moves. It could be, as Green et al. suggest, that the tephritid mimicry evolved from courtship

\title{
Infrared excess stirs cosmologists
}

ELECTROMAGNETIC radiation of all wavelengths fills the Universe. The 3-K microwave background is the best known and most studied cosmological emission, but in the past decade or so, sophisticated (often airborne) instruments have enabled astronomers to measure the temperature of the heavens at almost every wavelength. Much of this radiation is simply accumulated emission from our own Galaxy, but there are a few windows in the spectrum through which the Universe can be glimpsed. At a recent meeting*, signs of an excess of infrared at two different wavelengths were reported, and theoretical cosmologists were quick to hang their own theories on the proffered pegs.

T. Matsumoto (Nagoya University) described results from an infrared detector put above the interfering atmosphere by rocket. Such experiments are both technically difficult, and hard to interpret theoretically. The detector must be cooled, usually by liquid helium, so that thermal emission from the instrument itself does not overwhelm the tiny cosmological signal. And the detected signal itself will usually be dominated by radiation from various local sources: integrated starlight, zodiacal light, emission from interplanetary dust, and so on. All this must be independently measured and subtracted before any putative cosmological radiation is revealed.

Matsumoto first drew attention to measurements in the near infrared (wavelengths of a few micrometres), and claimed that there was evidence for a small excess at about $2 \mu \mathrm{m}$. On this point the audience seemed generally unconvinced; most participants were inclined to think that a small adjustment in the various subtractions could easily account for the supposed signal. But Matsumoto also showed results from a more recent flight, in which there *Comets to Cosmology, 3rd International IRAS Conference, London, 6-10 July 1987.

display; but one has the uncomfortable feeling that it simply is courtship display, and that its anti-jumping spider effect is no more than a happy accident.

Of course the display might have both functions, and if jumping spiders do exert strong predation pressure in the wild, as Green et al. assert, then there is every reason for courtship displays to take on the dual-purpose role. If it can be inferred that this behaviour is genuinely protective, then it does seem to be mimicry of a new kind. The converse type, where the predator mimics its prey, is well known; perhaps the best example is the firefly Phocturus versicolor, where the female lures males of other species to their fate by imitating the flash patterns of their respective females (Lloyd, J.E. Scient. Am. 245(1), 138-145; 1981). Here we have the reverse, a prey species that gains by was credible evidence for an excess in the far infrared, at a wavelength of a little less than $1 \mathrm{~mm}$. For this wavelength, at a higher energy than the peak of the $3-\mathrm{K}$ background and less than the characteristic 20-K emission from galactic molecular clouds, the subtraction is easier, and the proposed cosmological origin of the excess seems the most likely explanation.

Where does the signal come from? P. L. Richards (Berkeley), talking about the microwave background, said unequivocally that there was now no evidence for any distortion from an exactly thermal spectrum at $2.7 \mathrm{~K}$. But Matsumoto's signal would correspond to a component of thermal emission at 3.1 K. Other explanations all attribute the excess to some kind of comparatively recent astrophysical process. In fact, as B. J. Carr (Queen Mary College) pointed out, it is not hard to think of explanations, and therefore Matsumoto's signal is of little help in distinguishing one cosmological model from another. The most prosaic origin is that the $1-\mathrm{mm}$ excess is a redshifted remnant of the galactic $20-K$ emission which is prominent today; a population of early galaxies at high redshift is not difficult to find.

Slightly more exotic is the notion that a pregalactic generation of extremely massive stars, evolving on a very short-time scale, would leave behind a suitable radiation signal. In both these cases, care must be taken to ensure that the proposed source does not exceed observed emission levels at other wavelengths, but this constraint is neatly avoided in the most exotic explanation, espoused by J. Ostriker: a population of superconducting strings, whose main purpose is to initiate galaxy formation, produces radiation of a characteristic energy. The string population and its redshift can be chosen to give Matsumoto's infrared excess, and there is no significant signal elsewhere.

David Lindley

\section{imitating its predator.}

The effectiveness of leg mimicry provides convincing, if circumstantial, evidence that leg patterns are indeed the main sign stimuli used by the jumping spiders to distinguish prey from conspecifics. I have made an observation that further supports this view, which is that on occasion jumping spiders mimic themselves. The figure shows a male of Metaphidippus imperialis, a species inhabiting Californian redwood forests where there is too little light for the black legs themselves to be seen properly. Presumably, the leg-like pattern on the face has the same function as the tetriphids' wing patterns: to convince other jumping spiders that this is indeed one of their own kind.

M.F. Land is Professor of Neurobiology in the School of Biological Sciences, University of Sussex, Brighton BN1 9QG, UK. 